ARTICLE

\title{
Modeling Radiation Chemistry in the Geant4 Toolkit
}

\author{
Mathieu KARAMITROS ${ }^{1}$, Alfonso MANTERO ${ }^{2}$, Sébastien INCERTI ${ }^{1,{ }^{*}}$, Werner FRIEDLAND ${ }^{3}$, \\ Gérard BALDACCHINO ${ }^{4}$, Philippe BARBERET ${ }^{1}$, Mario BERNAL ${ }^{5}$, Riccardo CAPRA ${ }^{6}$, Christophe CHAMPION ${ }^{1,7}$, \\ Ziad EL BITAR $^{8}$, Ziad FRANCIS ${ }^{9}$, Paul GUEेYE ${ }^{10}$, Anton IVANCHENKO ${ }^{1}$, Vladimir IVANCHENKO ${ }^{7,11}$, \\ Hisaya KURASHIGE ${ }^{12}$, Barbara MASCIALINO ${ }^{13}$, Philippe MORETTO ${ }^{1}$, Petteri NIEMINEN ${ }^{14}$, Giovanni SANTIN ${ }^{14}$, \\ Hervé SEZNEC ${ }^{1}$, Hoang N. TRAN ${ }^{1}$, Carmen VILLAGRASA ${ }^{9}$ and Christina ZACHARATOU ${ }^{15}$ \\ ${ }^{1}$ Université Bordeaux 1, CNRS/IN2P3, Centre d'Etudes Nucléaires de Bordeaux Gradignan, CENBG, \\ Chemin du Solarium, BP120, 33175 Gradignan, France \\ ${ }^{2}$ INFN Sezione di Genova, Via Dodecaneso 33,16146 Genova, Italy \\ ${ }^{3}$ Institute of Radiation Protection, Helmholtz Zentrum München, German Research Center for Environmental Health, \\ Ingolstaedter Landstr. 1, 85764 Neuherberg, Germany \\ ${ }^{4}$ CEA Saclay, IRAMIS, UMR 3299 CEA-CNRS SIS2M, Laboratoire de Radiolyse, F-91191 Gif-sur-Yvette Cedex, France \\ ${ }^{5}$ Instituto de Física Gleb Wataghin, Universidade Estadual de Campinas-UNICAMP, SP 13083-859, Brazil \\ ${ }^{6}$ Via Niella 12, 17100 Savona, Italy \\ ${ }^{7}$ Laboratoire de Physique Moléculaire et des Collisions, Université Paul Verlaine-Metz, \\ 1 Boulevard Arago,Technopôle 2000, 57078 Metz, France \\ ${ }^{8}$ Institut Pluridisciplinaire Hubert CURIEN, 23 rue du loess, BP28, 67037 Strasbourg cedex 2, France \\ ${ }^{9}$ IRSN, Institut de Radioprotection et de Sûreté Nucléaire, BP17, 92262 Fontenay-aux-Roses, France \\ ${ }^{10}$ Department of Physics, Hampton University, Hampton, Virginia 23668, USA \\ ${ }^{11}$ Ecoanalytica, 119899 Moscow, Russia \\ ${ }^{12}$ Kobe University, Japan \\ ${ }^{13}$ Department of Medical Radiation Physics, Stockholm University, P.O. Box 260, SE-171 76 Stockholm, Sweden \\ ${ }^{14}$ ESA-ESTEC, 2200 AG Noordwijk, The Netherlands \\ ${ }^{15}$ Niels Bohr Institute, Blegdamsvej 17, 2100 Copenhagen, Denmark
}

On behalf of the Geant4 Standard and Low-Energy Electromagnetic Physics Working Groups. The authors are members of the Geant4-DNA collaboration.

\begin{abstract}
Simulation of biological effects of ionizing radiation at the DNA scale requires not only the modeling of direct damages induced on DNA by the incident radiation and by secondary particles but also the modeling of indirect effects of radiolytic products resulting from liquid water radiolysis. They can provoke single, double strand breaks and base damage by reacting with DNA. The Geant4 Monte Carlo toolkit is currently being extended for the simulation of biological damages of ionizing radiation at the DNA scale in the framework of the "Geant4-DNA" project. Physics models for the modeling of direct effects are already available in Geant4. In the present paper, an approach for the modeling of radiation chemistry in pure liquid water within Geant4 is presented. In particular, this modeling includes Brownian motion and chemical reactions between molecules following water radiolysis. First results on time-dependent radiochemical yields from 1 picosecond up to 1 microsecond after irradiation are compared to published data and discussed.
\end{abstract}

KEYWORDS: Geant4, Geant4-DNA, DNA, microdosimetry, liquid water, chemistry, radiolysis, radiobiology, radiation damage

\section{Introduction}

The accurate modeling of interactions of ionizing radiation with biological matter remains a challenge in radiobiology. Monte Carlo simulation techniques can provide solutions allowing for example the prediction of biological DNA damages, such as single, double strand break and base damages, which can be measured experimentally. Such damages may directly impact on the cell mutation, quiescence or death. The Geant 4 Monte Carlo simulation toolkit, an open source set of object-oriented libraries developed for modeling the passage of particles

*Corresponding author, E-mail: incerti@cenbg.in2p3.fr

(c) 2011 Atomic Energy Society of Japan, All Rights Reserved. through matter, covers a large variety of application domains, ranging from high energy physics to space and medical applications. In particular, the Geant4-DNA project, ${ }^{1,2}$ an activity of the Geant 4 collaboration, aims at providing Geant4 with new open source capabilities specific to radiobiology applications, including the modeling of elementary physical interactions down to the sub-electronVolt scale, liquid water radiolysis and radiation chemistry. The project focuses primarily on the prediction of early direct and non-direct DNA damages up to $1 \mu$ s after irradiation.

Advanced simulation codes have already been developed by several groups for the modeling of ionizing radiation damages on the DNA molecule. They include radiation che- 
mistry modeling (e.g., the PARTRAC software, ${ }^{3,4,5)}$ the work of S. Uehara and H. Nikjoo ${ }^{6)}$ and the RADACK software ${ }^{7)}$ ) and some of them also include a detailed DNA geometrical model in order to study DNA strand damages and structure modification. A detailed review of these codes is available $\mathrm{in}^{8)}$. However, these codes are usually not easily accessible to users and they are often designed for very specific applications which can limit their applicability domain. As an alternative, the Geant4-DNA project is based on the general purpose Geant 4 Monte Carlo toolkit which offers to users a rich set of electromagnetic, optical and hadronic physics processes from the electronVolt range up to a few $\mathrm{GeV} / \mathrm{n}$, which can be combined in a multi-scale approach. ${ }^{9)}$ In parallel Geant4 proposes sophisticated geometry modeling capabilities for example recently applied from the scale of biological cells to the scale of planets. ${ }^{10-11)}$ The platform is available freely on the Internet for several computing environments. $^{12)}$

Potential applications of the Geant4-DNA project are foreseen for long duration manned space exploration programs as well as for the use of radiation in the medical domain (e.g., particle therapy) where radiation effects at the cellular level are of concern.

This work presents the first version of a prototype software developed in the framework of the Geant4-DNA project for the modeling of liquid water radiation chemistry in the Geant 4 toolkit. The aim of this prototype is to demonstrate the possibility to deliver to the scientific community such modeling capabilities through a general purpose and open source Monte Carlo simulation toolkit.

\section{Principle of Radiation Chemistry Simulation}

One of the potential applications of radiation chemistry simulations for cellular radiobiology is to evaluate the ratio between DNA strand breaks created by primary and secondary ionizing particles directly on the DNA molecule (the so-called "direct effects") and those provoked by reactive oxygen species (the so-called "indirect effects") produced through water radiolysis by incident ionizing radiations on the biological medium, mainly liquid water.

After ionization or excitation by a primary or secondary particle, a water molecule can dissociate into new molecules. These new molecular species can either interact amongst themselves, producing other molecules, or react with the DNA molecule and finally induce DNA damages. Consequently, after the physical irradiation, the number of molecules of a given species in liquid water changes with time.

In order to verify the reliability of such a simulation, the time-evolution of the number of molecules in liquid water (without simulating explicitly the DNA molecule itself) can be compared to other theoretical predictions. For a given molecular species, this evolution depends on the molecules' capacity to diffuse into the medium (pure liquid water in our case) and depends also on the proximity of the molecules with their respective potential reactants.

\section{Scenario of Radiation Chemistry in Liquid Wa- ter}

The stochastic Monte Carlo simulation of early biological damages on the DNA molecule from ionizing radiation follows several identified consecutive stages, as explained globally in References 4, 7 and 13.

\section{The Physical Stage}

The first stage following cellular irradiation is the so-called physical stage. In this stage, all physical interactions take place. For e.g. in the case of electrons, all the elastic and inelastic interactions are simulated with Geant4-DNA, namely, the elastic scattering, the ionization and the electronic excitation.

\section{The Physico-Chemical Stage}

From 1 femtosecond (fs) to 1 picosecond (ps), the physico-chemical stage encompasses the very fast events (mainly electronic), such as thermalization and solvation of subexcitation electrons, electronic hole migration and fast electronic recombination that leads to chemical bond breaks. For the prototype development reported in this paper, we follow the approach proposed by PARTRAC, ${ }^{4,5)}$ where branching ratios and thermalization distances are the same for any incoming particle and energy.

Excited water molecules are assumed to decay at $100 \mathrm{fs}$ after irradiation. ${ }^{13)}$

The electronic holes of ionized water molecules quickly migrate from a molecule to another. This process lasts about 0.5 fs. $^{14,15)}$

At $10 \mathrm{fs}$ after the electron removal, ${ }^{16)}$ a proton transfer occurs between the final ionized water molecule and a nearby water molecule to originate $\mathrm{H}_{3} \mathrm{O}^{+}$and $\cdot \mathrm{OH}$ molecules from the reaction: $\mathrm{H}_{2} \mathrm{O}^{+}+\mathrm{H}_{2} \mathrm{O} \rightarrow \mathrm{H}_{3} \mathrm{O}^{+}+\cdot \mathrm{OH}$.

The secondary electrons produced during the physical stage get thermalized within a characteristic time of $110 \mathrm{fs}$ through vibrational excitation of water and they become solvated within a characteristic time of $250 \mathrm{fs}{ }^{17)}$ In our simulation, the subexcitation electrons can undergo dissociative attachment, vibrational excitation and elastic scattering processes until they reach $25 \mathrm{meV}$. Afterwards, we consider them as solvated and they start to diffuse through Brownian motion.

The hot dissociation fragments of water molecules are assumed to become all thermalized within $1 \mathrm{ps}$. At $1 \mathrm{ps}$, the placement of the fragments from the hit mother water molecule can drastically affect the simulated radiochemical yields. If, for example in the case of a water molecule in the A1B1 excitation state, the dissociation fragments $\mathrm{H} \bullet$ and $\bullet \mathrm{OH}$ are placed too close from each other, they would rapidly recombine into $\mathrm{H}_{2} \mathrm{O}$.

\section{The Chemical Stage}

From 1 ps to 1 microsecond ( $\mu \mathrm{s})$, in the so-called chemical stage, the molecular species can diffuse through the medium and interact with each other or with the DNA mo- 
lecule. The diffusion process and mutual interactions are described in more details in Section V.

\section{Software Requirements}

In order to simulate radiation chemistry of liquid water in Geant4, the software fulfills the following five requirements (SR):

- SR\#1: description and management of molecules, including some of their static (name, number of atoms, ground state configuration, decay table...) and dynamic (electronic configuration, diffusion coefficient...) properties;

- SR\#2: identification of the electronic state of excited and ionized water molecules in the corresponding Geant4-DNA physics models;

- SR\#3: decay process for molecules following excitation and ionization, including thermalization;

- SR\#4: diffusion process for molecules according to Brownian motion;

- SR\#5: chemical interaction process between diffusing molecules;

Geant4 9.4 does not currently include any class for handling molecules. With this prototype, it is the first time that Geant 4 is extended to track molecules and model mutual interactions between them. Note that the prototype software described in this work is compatible with Geant 49.4 release and will be delivered publicly with default input parameters for the modeling of water radiolysis.

\section{Molecular Species and Chemical Processes}

Under ionizing radiation, excited and ionized water molecules may decay and dissociate into new molecules $\left(\mathrm{e}_{\mathrm{aq}}^{-}\right.$, $\mathrm{H}_{2}, \mathrm{H} \bullet, \bullet \mathrm{OH}, \mathrm{H}_{3} \mathrm{O}^{+}$) which can diffuse and interact mutually to produce other molecules $\left(\mathrm{OH}^{-}, \mathrm{H}_{2} \mathrm{O}_{2}\right)$. These $\mathrm{e}_{\mathrm{aq}}^{-}, \mathrm{H} \bullet$, - $\mathrm{OH}$ and $\mathrm{H}_{2} \mathrm{O}_{2}$ species can also directly interact with DNA components.

New prototype processes in Geant4 for liquid water radiolysis and the resulting chemistry have been developed in Geant4 in the framework of the Geant4-DNA project. We have adopted the approach followed by the state-of-the-art Monte Carlo PARTRAC software, which is described in detail by their authors in the literature. ${ }^{4,5)}$ Consequently, in this work, we pay a particular attention to the time-dependent radiochemical yields calculated with PARTRAC. The adopted software design was made flexible enough to allow in the future the inclusion of alternative radiochemistry models.

With the present software prototype, the chemical stage can be simulated up to $1 \mu$ s after irradiation. The simulation starts with the positions of radiolytic products of the water molecules at 1 ps after the physical irradiation, using the branching ratios adjusted by PARTRAC. ${ }^{4}$

\footnotetext{
${ }^{\mathrm{a}} \mathrm{e}_{\mathrm{aq}}^{-}$: a solvated electron in water (also named aqueous solvated electron) is a free electron in an aqueous solution surrounded by a "shield" of water molecules.
}

The hot products have reached a so-called "thermalization distance" from the hit mother water molecule before starting a Brownian motion. The method adopted to simulate the thermalization process in this work can be found in. ${ }^{4)}$ The flexibility of the prototype software allows the user to easily implement his/her own thermalization distance computation method for each decay channel.

Brownian diffusion of radiolytic products uses a random change of direction after a time step $\Delta t$, corresponding to a geometrical mean step size given by:

$$
\langle R\rangle=\sqrt{6 . D \cdot \Delta t}
$$

where $D$ is the diffusion coefficient. The diffusion coefficient depends on the molecular species and on its excited state, while the time step can be selected as a function of the physical time.

The criterion for the chemical reactions to occur is the physical distance between two molecules. If two molecules are closer than a calculated reaction radius, ${ }^{18)}$ related to the chemical reaction rate, a reaction is assumed to happen, using a jump-through correction. ${ }^{18)}$

\section{Simulation Parameters}

The default branching ratios (Table 1), diffusion coefficients (Table 2), reaction rates (Table 3) and time steps $(\Delta t)$ (Table 4) of this prototype software are those proposed by PARTRAC, ${ }^{4)}$ but the user still has the possibility to easily define his/her own parameters and models.

Table 1 Branching ratios of a water molecule at 1 ps as described in Reference 4

\begin{tabular}{ccc}
\hline Electronic state & Decay Channel & Fraction (\%) \\
\hline All ionization states & $\mathrm{H}_{3} \mathrm{O}^{+}+\bullet \mathrm{OH}$ & 100 \\
\hline Excitation state & $\bullet \mathrm{OH}+\mathrm{H} \bullet$ & 65 \\
A1B1: & $\mathrm{H}_{2} \mathrm{O}+\Delta \mathrm{E}$ & 35 \\
$(1 \mathrm{~b} 1) \rightarrow(4 \mathrm{a} 1 / 3 \mathrm{~s})$ & & \\
\hline Excitation state & $\mathrm{H}_{3} \mathrm{O}^{+}+\bullet \mathrm{OH}+\mathrm{e}_{\text {aq }}^{-}$ & 55 \\
B1A1: & $\bullet \mathrm{OH}^{+} \bullet \mathrm{OH}+\mathrm{H}_{2}$ & 15 \\
$(3 \mathrm{a} 1) \rightarrow(4 \mathrm{a} 1 / 3 \mathrm{~s})$ & $\mathrm{H}_{2} \mathrm{O}+\Delta \mathrm{E}$ & 30 \\
\hline Excitation state: & $\mathrm{H}_{3} \mathrm{O}^{+}+\bullet \mathrm{OH}+\mathrm{e}_{\text {aq }}^{-}$ & 50 \\
Rydberg, diffusion & $\mathrm{H}_{2} \mathrm{O}+\Delta \mathrm{E}$ & 50 \\
bands & & \\
\hline
\end{tabular}

Table 2 Diffusion coefficients for the diffusing species as described in Reference 4

\begin{tabular}{cc}
\hline Species & Diffusion coefficient D $\left(10^{-9} \mathrm{~m}^{2} \mathrm{~s}^{-1}\right)$ \\
\hline $\mathrm{e}_{\mathrm{aq}}^{-}$ & 4.9 \\
$\bullet \mathrm{OH}$ & 2.8 \\
$\mathrm{H} \bullet$ & 7.0 \\
$\mathrm{H}_{3} \mathrm{O}^{+}$ & 9.0 \\
$\mathrm{H}_{2}$ & 4.8 \\
$\mathrm{OH}^{-}$ & 5.0 \\
$\mathrm{H}_{2} \mathrm{O}_{2}$ & 2.3 \\
\hline
\end{tabular}


Table 3 Reaction rates as described in Reference 4

\begin{tabular}{|c|c|}
\hline Reaction & Reaction rate $\left(10^{10} \mathrm{M}^{-1} \mathrm{~s}^{-1}\right)$ \\
\hline $\mathrm{H} \cdot+\mathrm{e}_{\mathrm{aq}}^{-}+\mathrm{H}_{2} \mathrm{O} \rightarrow \mathrm{OH}^{-}+\mathrm{H}_{2}$ & 2.65 \\
\hline $\mathrm{H} \bullet+\bullet \mathrm{OH} \rightarrow \mathrm{H}_{2} \mathrm{O}$ & 1.44 \\
\hline $\mathrm{H} \bullet+\mathrm{H} \bullet \rightarrow \mathrm{H}_{2}$ & 1.20 \\
\hline $\mathrm{H}_{2}+\bullet \mathrm{OH} \rightarrow \mathrm{H} \bullet+\mathrm{H}_{2} \mathrm{O}$ & $4.17 \times 10^{-3}$ \\
\hline $\mathrm{H}_{2} \mathrm{O}_{2}+\mathrm{e}_{\mathrm{aq}}^{-} \rightarrow \mathrm{OH}^{-}+\bullet \mathrm{OH}$ & 1.41 \\
\hline $\mathrm{H}_{3} \mathrm{O}^{\top}+\mathrm{e}_{\mathrm{aq}}^{-} \rightarrow \mathrm{H} \bullet+\mathrm{H}_{2} \mathrm{O}$ & 2.11 \\
\hline $\mathrm{H}_{3} \mathrm{O}^{\top}+\mathrm{OH}^{-} \rightarrow 2 \mathrm{H}_{2} \mathrm{O}$ & 14.3 \\
\hline$\bullet \mathrm{OH}+\mathrm{e}_{\mathrm{aq}}^{-} \rightarrow \mathrm{OH}^{-}$ & 2.95 \\
\hline$\cdot \mathrm{OH}+\cdot \cdot \mathrm{OH} \rightarrow \mathrm{H}_{2} \mathrm{O}_{2}$ & 0.44 \\
\hline $\mathrm{e}_{\mathrm{aq}}^{-}+\mathrm{e}_{\mathrm{aq}}^{-}+2 \mathrm{H}_{2} \mathrm{O} \rightarrow 2 \mathrm{OH}^{-}+\mathrm{H}_{2}$ & 0.50 \\
\hline
\end{tabular}

Table 4 Time steps $\Delta t$ with respect to the physical time, as described in Reference 4

\begin{tabular}{lc}
\hline \multicolumn{1}{c}{ Time interval (s) } & $\Delta t(\mathrm{ps})$ \\
\hline Until $10^{-11}$ & 0.1 \\
$10^{-11}-10^{-10}$ & 1 \\
$10^{-10}-10^{-9}$ & 3 \\
$10^{-9}-10^{-8}$ & 10 \\
Above $10^{-8}$ & 100 \\
\hline
\end{tabular}

\section{Time-Dependent Radiochemical Yields}

For a given molecular species, the time-dependent radiochemical yield $G$ is defined as the number of molecules produced for a total absorbed energy of $100 \mathrm{eV}$ in the irradiated medium:

$$
G=\frac{N(t) \times 100}{E(e V)},
$$

where $N(t)$ is the number of molecules and $E$ is the total energy deposit by the incident ionizing particle into the medium, expressed in $\mathrm{eV}$.

Experimental data on time-dependent chemical yields are rare and the simulations often do not reproduce the exact conditions of the experimental set-up (type and energy of the incoming particle, volume of the target). However, in order to fill in this lack, most authors compare their simulated results with experiments at similar LET value.

In order to verify our prototype software, we compare our results with other simulation codes (PARTRAC ${ }^{4,5)}$ and Uehara's and Nikjoo's work ${ }^{6)}$ ). However, as Geant4-DNA and PARTRAC ${ }^{4,5,19)}$ physics models are not identical, the outcome of the physical stage in the two simulations will necessarily differ. The physics models adopted in Geant4-DNA are fully described in Reference 2.

The presented results were obtained using the above default parameters, by shooting $2.5 \times 10^{4}$ incident electrons of $1 \mathrm{MeV}$ and considering only the first $10 \mathrm{keV}$ lost by the primary track, as done in PARTRAC ${ }^{4)}$ and Uehara's and Nikjoo's work. ${ }^{6)}$ All secondary particles were tracked.

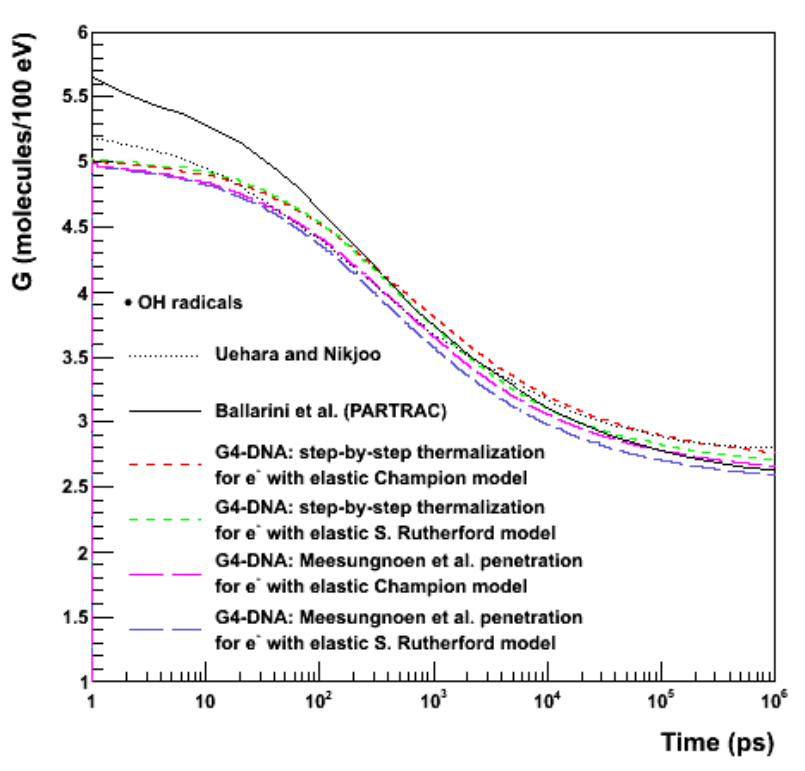

Fig. 1 Prototype results on $\cdot \mathrm{OH}$ radiochemical yields (molecules $/ 100 \mathrm{eV}$ ) from $1 \mathrm{MeV}$ incident electrons with respect to time (in picosecond). References: Ballarini et al. (PARTRAC), ${ }^{3)}$ S. Uehara and H. Nikjoo. ${ }^{6)}$

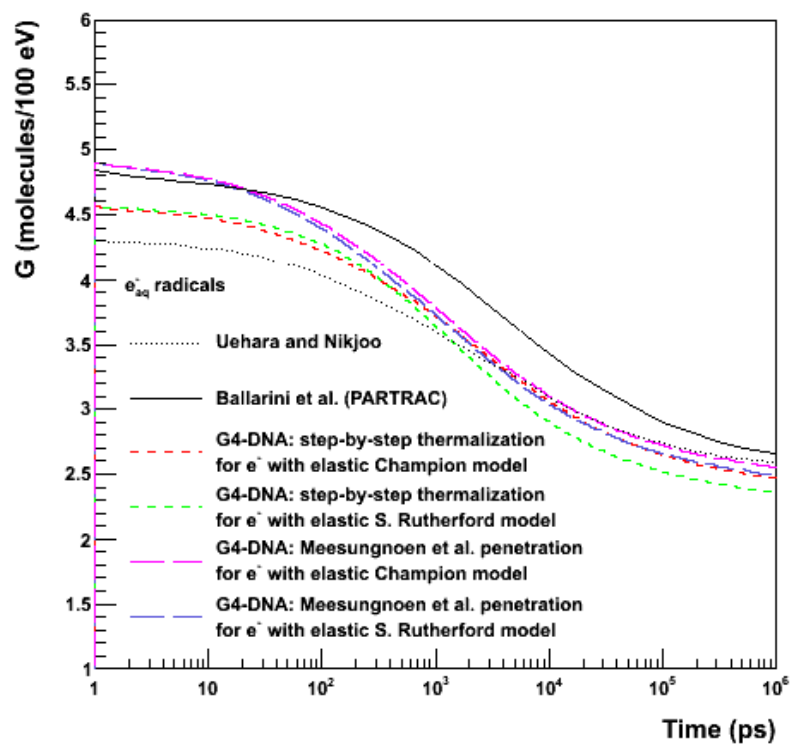

Fig. 2 Prototype results on $\mathrm{e}_{\mathrm{aq}}^{-}$radiochemical yields (molecules $/ 100 \mathrm{eV}$ ) from $1 \mathrm{MeV}$ incident electrons with respect to time (in picosecond). References: Ballarini et al. (PARTRAC), ${ }^{3)}$ S. Uehara and H. Nikjoo. ${ }^{6)}$

The resulting radiochemical yields for $\bullet \mathrm{OH}$ and solvated electrons are shown in Figs. 1 and $\mathbf{2}$ respectively. These preliminary results have been obtained using the following Geant4-DNA processes (the corresponding Geant4-DNA models are indicated in parenthesis): electron elastic scatter- 
ing (Champion model or Screened Rutherford model), electronic excitation (Born excitation model), ionization (Born ionization model), vibrational excitation (Sanche model) and dissociation attachment (Melton model). These processes and models are available in the Geant 4 release 9.4 and they are described in References 2 and 20.

The results of this prototype software are compared to other theoretical calculations. The computation of the radiochemical yields was performed using two different computation methods for the tracking of very low energy electrons. In the first approach, we followed the electrons step-by-step down to $25 \mathrm{meV}$ using the processes mentioned above. In the second approach, we used the penetration for low energy electrons computed by Meesungnoen et al.: ${ }^{21)}$ when the electron reaches a kinetic energy inferior to the lowest excitation level $(8.22 \mathrm{eV})$, it is displaced in one unique and final step whose mean value corresponds to a fit of the penetration curve given $i^{21)}$ and with an isotropic direction. In the two approaches described above, electron elastic scattering was computed using either the Champion model or the Screened Rutherford model. ${ }^{2)}$

Compared to PARTRAC, Geant4-DNA early yield for hydroxyl radicals appears slightly underestimated. The use of different physics models between PARTRAC and Geant4-DNA could account for this difference. One can also note that the dissociation scheme of B1A1 excitation channel is slightly different in Ballarini et al. ${ }^{3)}$ with respect to that of Kreipl et al. ${ }^{4)}$

On the other hand, radiochemical yields at 1 microsecond for both solvated electrons and hydroxyl radicals appear to converge in all simulations. However, one can notice that the step-by-step tracking of very low energy electrons leads to smaller G-values than the approach which uses the one step placement of the electron. The slight shift observed at 1 ps for solvated electrons can be explained considering that below the excitation threshold, the electron interacts with the liquid water medium through three processes: elastic scattering, dissociative attachment and vibrational excitation. In the step-by-step approach, the electron can undergo dissociative attachment down to $4 \mathrm{eV}$, which depresses the number of solvated electron present at one picosecond. However, in the second approach, no absorption of the electron via dissociative attachment occurs below the lowest excitation energy.

Furthermore, the thermalization distance computed at $7 \mathrm{eV}$ via the step-by-step approach with the Champion (Screened Rutherford) elastic model is about 40\% (60\%) lower than the Meesungnoen's approach. As a result, in the first approach, the secondary electrons are closer to the water molecule they come from and thus closer to the products of dissociation. As a consequence, in the case of full tracking of low energy electrons, albeit there are less solvated electrons at 1 picosecond (because they are captured by water molecules), reactions are more likely to occur at earlier times due to their smaller computed range during the physics stage.

Although the comparison of PARTRAC physics models with Geant4-DNA ones is out of the scope of this contribu- tion, we have verified as an illustration that a systematic $100 \%$ increase of the electron cross sections for ionization and excitation leads to no significant increase of $G$ values at $1 \mathrm{ps}$ and to a $10 \%$ increase on both yields at $1 \mu \mathrm{s}$ in the described conditions.

\section{Conclusion}

A first software prototype dedicated to the simulation of liquid water radiation chemistry has been developed within the general purpose and open source Geant4 Monte Carlo simulation toolkit, in the framework of the Geant4-DNA project. This prototype demonstrates the feasibility of such modeling in Geant4 and will allow to users to benefit fully from Geant 4 capabilities and features. The comparison to other liquid water radiation chemistry simulations, although highlighting some differences, shows a qualitatively good agreement. The described prototype software is still under development and uses recent models presented in Reference 20 which are included in the Geant 49.4 release. It will be fully released publicly in the near future and will be validated against experimental data. We also expect to compare our results to dedicated radiolysis experiments that will be performed at the Laboratoire de Radiolyse (CEA Saclay, Gif-sur-Yvette, France) in order to measure time-dependent - $\mathrm{OH}$ yields by using chemiluminescence technics. ${ }^{22,23)}$

\section{Acknowledgment}

We are grateful to Dr Marie Davidková (Nuclear Physics Institute AS CR, Praha, Czech Republic) for her advice and suggestions and also for providing us with relevant publications. We are also thankful to Dr Makoto Asai (SLAC National Accelerator Laboratory, Menlo Park, CA, USA), spokesperson of the Geant4 collaboration, for his technical advice. The Geant4-DNA project receives funding from the French Agence Nationale de la Recherche Contract No. ANR-09-BLAN-0135-01 and from the European Space Agency under Contract No. AO6041-22712/09/NL/AT. The work was also supported in part by the RFBR grant 09-02-91065 and the CNRS grant PICS-4865.

\section{References}

1) S. Incerti et al., "The Geant4-DNA project," Int. J. Model. Simul. Sci. Comput., 1[2], 157-178, (2010).

2) S. Incerti et al., "Comparison of GEANT4 very low energy cross section models with experimental data in water," Med. Phys, 37[9], 4692-4708, (2010).

3) F. Ballarini et al., "Stochastic aspects and uncertainties in the prechemical and chemical stages of electron tracks in liquid water: a quantitative analysis based on Monte Carlo simulations," Radiat. Environ. Biophys., 39[3], 179-188, (2000).

4) M. S. Kreipl, W. Friedland, H. G. Paretzke, "Time- and space-resolved Monte Carlo study of water radiolysis for photon, electron and ion irradiation," Radiat. Environ. Biophys., 48[1], 11-20, (2009).

5) W. Friedland et al., "Track structures, DNA targets and radiation effects in the biophysical Monte Carlo simulation code PARTRAC," Mutat. Res.: Fundam. Mol. Mech. Mutagen., doi:10.1016/j.mrfmmm.2011.01.003, (2011). 
6) S. Uehara, H. Nikjoo, "Monte carlo simulation of water radiolysis for low-energy charged particles," J. Radiat. Res., 47[1], 69-81, (2006).

7) V. Michalik, M. Begusová, E. A. Bigildeev, "Computer-aided stochastic modeling of the radiolysis of liquid water," Radiat. Res., 149[3], 224-236, (1998).

8) H. Nikjoo et al., "Track-structure codes in radiation research," Radiat. Meas., 41[9-10], 1052-1074, (2006).

9) V. Ivantchenko et al., "Recent improvements in Geant4 electromagnetic physics models and interfaces," Proc. Joint Int. Conf. on Supercomputing in Nuclear Applications and Monte Carlo 2010 (SNA + MC2010), Tokyo, Japan, October 17-21, 2010 (2010).

10) S. Incerti et al., "Monte Carlo dosimetry for targeted irradiation of individual cells using a microbeam facility," Rad. Prot. Dos., 133[1], 2-11, (2009).

11) A. Le Postollec et al., "Monte-Carlo Simulation of the radiation environment encountered by a biochip during a mission to Mars," Astrobiology, 9[3], 311-323, (2009).

12) http://www.geant4.org, http://geant4.in2p3.fr/spip.php?rubrique14\&lang=en, https://twiki.cern.ch/twiki/bin/view/Geant4/LoweMigratedDN AProcesses

13) M. Davidkova, M. Spotheim-Maurizot, "Predicting radiation damage distribution in biomolecules," in Radiation chemistry : from basics to applications in material and life sciences, M. Spotheim-Maurizot, M. Mostafavi, T. Douki, J. Belloni (Ed.), EDP Sciences, Les Ulis, France, (2008).

14) A. Mozumder, J. L. Magee, "The early events of radiation chemistry," Int. J. Radiat. Phys. Chem., 7[2-3], 83-93, (1975).
15) H. Ogura, W. H. Hamill, "Positive hole migration in pulse-irradiated water and heavy water," J. Phys. Chem., 77[25], 2952-2954, (1973).

16) Y. Novakovskaya, "Theoretical estimation of the ionization potential of water in condensed phase. ii. superficial water layers," Prot. Met., 43[1], 22-33, (2007).

17) Y. Gauduel, S. Pommeret, A. Antonetti, "Femtosecond spectroscopy of ultrafast reactions in aqueous media," J. Phys. Condens. Matter, 2[S], SA171, (1990).

18) R. N. Hamm, J. E. Turner, M. G. Stabin, "Monte Carlo simulation of diffusion and reaction in water radiolysis - a study of reactant 'jump through' and jump distances," Radiat. Environ. Biophys., 36[4], 229-234, (1998).

19) M. Dingfelder et al., "Comparisons of calculations with PARTRAC and NOREC: transport of electrons in liquid water," Radiat. Res., 169[5], 584-594, (2008).

20) Z. Francis et al., "Molecular scale track structure simulations in liquid water using the Geant4-DNA Monte-Carlo processes," Appl. Radiat. Isot., 69[1], 220-226, (2011).

21) J. Meesungnoen et al., "Low-energy electron penetration range in liquid water," Radiat. Res., 158[5], 657-660, (2002).

22) V. Wasselin-Trupin et al., "A new method for the measurement of low concentrations of $\mathrm{OH} / \mathrm{O}_{2}{ }^{-}$radical species in water by high-LET pulse radiolysis. A time-resolved chemiluminescence study," J. Phys. Chem. A, 104[38], 8709-8714 (2000).

23) V. Wasselin-Trupin, G. Baldacchino, B. Hickel, "Detection of hydroxyl and superoxide radicals in radiolysis of water with time-resolved chemiluminescence," Can. J. Physiol. Pharmacol., 79[2], 171-175, (2001). 